\title{
Rapid Diagnosis of Malaria using Images of Stained Blood Smear
}

\author{
Dr. Narendra B. Mustare \\ Professor, CVR College of Engineering/EIE Department, Hyderabad, India \\ Email:drnamust@gmail.com
}

\begin{abstract}
This paper presents a technique to distinguish malaria parasite during blood checks recolored by Giemsa to improve the precision of distinguishing, on the preliminary step, the red platelet veil is extricated. It is owed toward the way that the big majority of malaria parasites live within pink platelets. At that factor, recolored components of blood, for example, pink platelets, parasites, and white platelets be extorted. On the subsequent level, pink platelet cowl is situated at the extracted recolored additives to isolate the potential parasites. By utilizing thick blood smear, quite a few blood smears can be analyzed hastily and no trouble in any respect. This work manages the programmed evaluation of parasite thickness in 'parasites according to a microliter of blood' from minuscule images of Giemsa-recolored thick blood smear. The principal factor of this examination is to look at the microscopic images of recolored dainty blood spreads using an assortment of processor apparition methods, reviewing malaria parasitemia on free factors (RBC's morphology). The projected process depends on the inductive method, shading of malaria parasites via versatile calculation of the Gaussian mixture model (GMM). Evaluation precision of RBCs is progressed, parting impediments of RBCs by separation alternate with close by maxima. Additionally, the characterization of tainted with non-contaminated RBCs has prepared for accurately evaluating parasitemia. Instruction along with assessment had completed on image dataset concerning floor facts, finding out the extent of infection with the affectability of $98 \%$. The exactness and skill ability of the proposed conspire about being programmed are demonstrated tentatively, outperforming other condition-of-the-art plans. Furthermore, this examination tended to the procedure with autonomous elements (RBCs' morphology).
\end{abstract}

Index Terms: first term, second term, third term, fourth term, fifth term, sixth term

\section{INTRODUCTION}

Malaria is considered one of the deadly international illnesses and can spark-off demise quickly on the off risk that its miles untreated. In 2015, 214 million instances of malaria were identified, which triggered around 438000 death [1]. Malaria is not unusual within tropical along with subtropical areas close to equator similar to Asia, SubSaharan Africa, and Latin America. It is impossible to resist malaria infection brought about through nonessential blood parasites of family Plasmodium. Malaria is Iran's main parasitic disease with a long background of episodes. Malaria fever is spread through the use of infected Anopheles female mosquitoes through their salivary glands that carry Plasmodium sporozoites. The kind Plasmodium has 4 organisms which can cause human distress: falciparum, vivax, ovale, and malaria. The famous process of life has arranged different development within the human and mosquito structure. The sporozoites join the process as a tainted mosquito blessing from the blood of an individual and transfer to the liver where they develop abiogenetically. Since 2010, WHO has advocated malaria disease symptomatic trying out by way of both mild microscopy and rapid demonstrative, [2,27]. RDT is applied wherein microscopy microscope is not on hand; this technique gives second outcomes however number one disservice of RDT is that parasite thickness cannot be assessed. The microscopy approach is the "Best remarkable level" for recognizing malaria parasites and assessing parasite thickness [2]. Varieties of blood films are arranged for minute localization: flimsy and smooth [2]. A small bloodstain is used to distinguish parasite types since the parasite identity is preserved. To distinguish the closeness of parasite and parasite thickness a dense bloodstain is introduced. A dense blood smear is more susceptible to multiple times than a thin blood smear, as it requires a greater amount of blood to be assessed [2]. $\square$

Malaria is generally diagnosed by manual microscopic examination of blood slide, which is named as "gold standard". However, the manual method of diagnosis is tedious, requires expert technicians, and prone to human errors. Besides, it also incurs a high cost for the training of technicians [6]. Considering these problems, the automatic analysis of the microscopic images for the diagnosis of malaria disease has gained importance. This motivated us to carry out this study to develop image-contrast enhancement and segmentation methods for rapid and accurate diagnosis of malaria through microscopic blood images [14].

\section{LITERATURE REVIEW}

Sathpathi et al., have proposed Microscopy of fringe blood slender with deep movies leftovers orientation for intestinal sickness conclusion. Even though Giemsa recoloring is most regularly utilized, the Leishman recoloring strategy gives a better perception of the atomic chromatin example of cells. It is less notable whether the exactness of parasitemia evaluation is similarly precise by the last strategy.

Naveed Abbas has proposed malaria parasitemia by the quantitative estimation of parasites within blood toward study level of disease. Light microscopy is a mainly excellent strategy used to study the blood for parasitemia assessment. Illustration evaluation of jungle fever parasitemia is relentless, tedious as well as emotional. Even though robotizing the procedure is a decent arrangement, the 
accessible strategies can't assess similar cases, for example, weakness and hemoglobinopathies because of deviation from ordinary RBCs' morphology.

Katherine Torres has proposed Microscopic assessment of Giemsa-recolored blood films stays a significant type of conclusion in malaria disease and is a reference standard for learning about. Nonetheless, similarly as with other perception-based conclusions, exactness relies upon singular professional execution, making institutionalization troublesome and dependability poor. Computerized image acknowledgment dependent on AI, using convolutional neural structures, gives the potential to conquer these disadvantages. A model automatic magnifying lens system utilizing a calculation depending on AI, the Autoscope, turned into evaluated for its capacity in malaria disease microscopy. In 2016, Autoscope became an effort at two fringe health offices in the Peruvian city of Iquitos, with repeated microscopy and PCR as guide norms. The important result measurements include the effect and accuracy of diagnosis commitment from Giemsa-recolored blood films, use PCR as a guide. $\square$

Ishan R.Dave, have created an endeavor in the preprocessing step to diminish varieties because of different components like lighting conditions and centralization of recoloring arrangement. The image is fragmented utilizing versatile thresholding, trailed by a few numerical morphological tasks. In the subsequent advance, different highlights dependent on shape, surface, shading, and recurrence space are separated. Utilizing the grouping step, the parasites are arranged into their right life stage or delegated leukocytes. The curiosity of the calculation is that it can recognize all the existence stages (ring, trophozoite, schizont, and gametocyte) of parasites and leukocytes [21]. It is not like identifying just ring life organize but is the best in class calculations. The inconsistency in the computerized parasite tally by the proposed calculation is $7.14 \%$, which is appropriate for PC based diagnosis (CAD) of malaria disease as indicated by world wellbeing association (WHO) quality control norms.

\section{Preprocessing \& Segmentation}

Right now, image is portioned into a frontal area (recolored particles for example parasites of all existence degrees, leukocytes, platelets, and numerous exclusive relics) and basis (nonstained particles as an example liaised purple platelets). Countless bogus high-quality (recolored gadgets) may come as locales of intrigue, to be wiped out in subsequent segments. The caught images are changed over in HSV color space to lessen sorts due to beauty and convergence of stain arrangement, which profoundly influences in RGB color space [4]. Stained and uncolored particles have a critical difference in their path of immersion. Owing to clamor, the picture immersion channel is divided by an ordinary channel of duration 3 along the way to reduce large variety. In Figure1, the histogram of direct immersion is considered. Since a large portion of the non-recolored molecule has poor channel appreciations for immersion, the investigation is done utilizing adaptive histogram thresholding of segmented particles from the immersion tube. As it appears in Figure1, the recolored portion of debris covers a small quarter of the histogram's $\mathrm{X}$-pivot. We can still observe that the recolored particles have a Cumulative Distribution Function (CDF) values greater than zero. Ninety-five after finding CDF. The stage of dimension is where $\mathrm{CDF}=0.95$ is taken. Using this Immersion channel picture at an edge level is binarized as shown in Equation beneath.

$$
\begin{array}{r}
\text { Pixel value }=\left\{\begin{array}{r}
1, \text { if saturation value }>T \\
0, \text { if saturation value }<T
\end{array}\right. \\
\text { where, } C D F(x=T)=0.95
\end{array}
$$

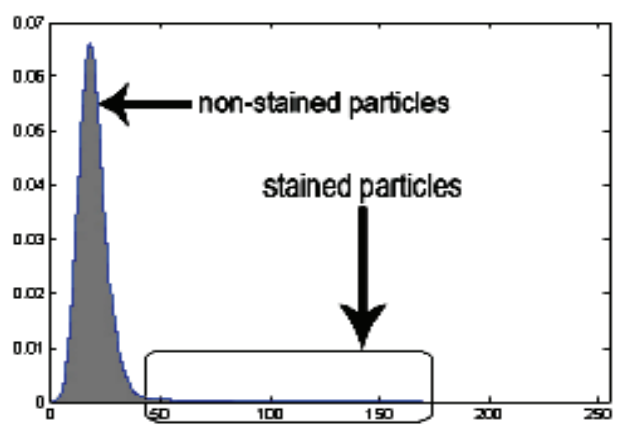

Figure 1. Histogram of saturation channel

The output of adaptive histogram thresholding is appeared in Figure 2(a). Numerical morphological tasks are utilized to remove the ideal segments from the portioned parallel image [2]. Openings are dispatched to kill little foundation regions inside the frontal area shape (Figure 2(b)). It is trailed by shutting activity to fill the little holes between segments of a similar molecule (Figure 2(c)). Shutting activity is performed utilizing a circle molded organizing component of range 3. Openings are filled again to take out foundation pixels inside closer view shapes (Figure 2(d)).
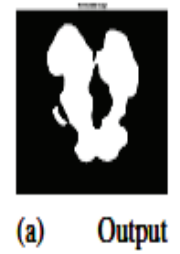
of adaptive thresholding

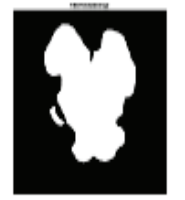

(b) Filled

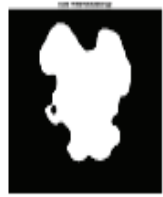

(c)

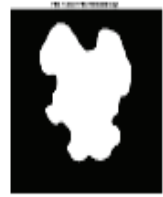

(d) +filled

Figure 2. Mathematical morphological operations

The yield after morphological tasks appears in Figure 3. 


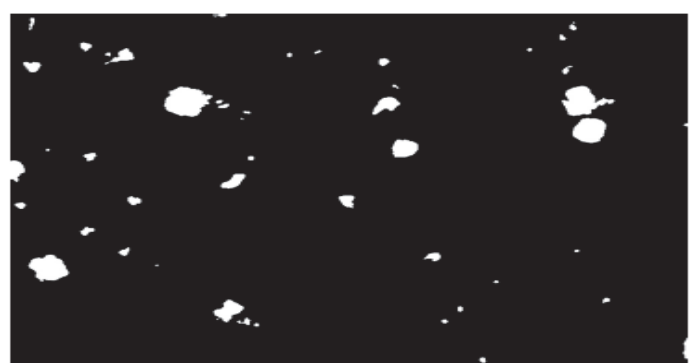

Figure 3. The output of prepossessing \& segmentation step.

\section{Proposed Methodology}

\section{A. Minuscule Image grabbing and preprocessing}

The critical point of our framework is to observe the perimeter blood spread and distinguish any parasitemia via utilizing image investigation on the procured pix. The securing of the image is a substantial increase within the complete technique. We make use of a compound first-rate discipline magnifying lens to filter the slides in-situ. This is done through and through the body under a trained pathologist's guidance. When collecting the image of the entire frame, the pathologist selects a particular area of interest (ROI). The chosen ROI is grabbed and distributed correctly through the analysis system [4, 25]. The use of morphology [8], Shading Correction [9], and wavelet techniques, primarily based on definite techniques, has had powerful effects on the identification of malaria parasites from blood smear pix. It is clear that the pictures obtained and sent are small in quality and are boisterous. Subsequently, we use de-noise, and the picture enhances techniques to configure the suitability of the dividing file. $\square$

We use the interscale symmetrical wavelet-based thing that is some other Stain's Objective Risk Estimator (SORE) way to cope with Unser et al-supported picture de-noise[6] for de-noise. The image histogram shows an amazing top; in the middle, we get wonderful results from using the Brightness Preserving Dynamic Histogram Equalization (BPDHE) measurement [16]. They will always remember at the department's computer and promise to go into the subtleties of the methodology of de-noise and enhancement right now.

\section{B. Shading Space Conversion}

Shading space transformation is the initial section in the imaging exam and carried out before running Normalized Cut (NCut) calculation for the mobile division. They are changing over the minute pics into extraordinary shading spaces (RGB to HSV, RGB to YCbCr and RGB to NTSC). Images are commonly placed away and showed inside the RGB area. Be that as it can, to guarantee the isotropy of the element area, a uniform shading area with the apparent shading contrasts anticipated with the aid of Euclidean separations have to be applied[5]. Here we are looking at the presentation of NCut calculation in 4 shading space (RGB, HSV, YCbCr, and NTSC).

\section{Division with Normalized Cut}

(Cap) the calculation above modified over shading area NCut be a solo division method that doesn't require an introduction and approaches the division issue as a chart dividing issue. NCut depends on a worldwide paradigm, and it expands both the complete divergence between the various gatherings and the absolute similitude inside the gatherings $[7]$.

$R G B$ : It is equipment situated model and is notable for its shading screen show reason. The NCuts on RGB appear empowering results yet it is likewise debased with loads of deceptive sections which make the framework not reasonable for computerized techniques.

$H S V$ : Tone $(\mathrm{H})$ is a shading quality that depicts an unadulterated shading, while immersion (S) characterizes the relative immaculateness or else sum of white light blended in by tone; esteem (V) alludes toward splendor of image as shown in figure (4).

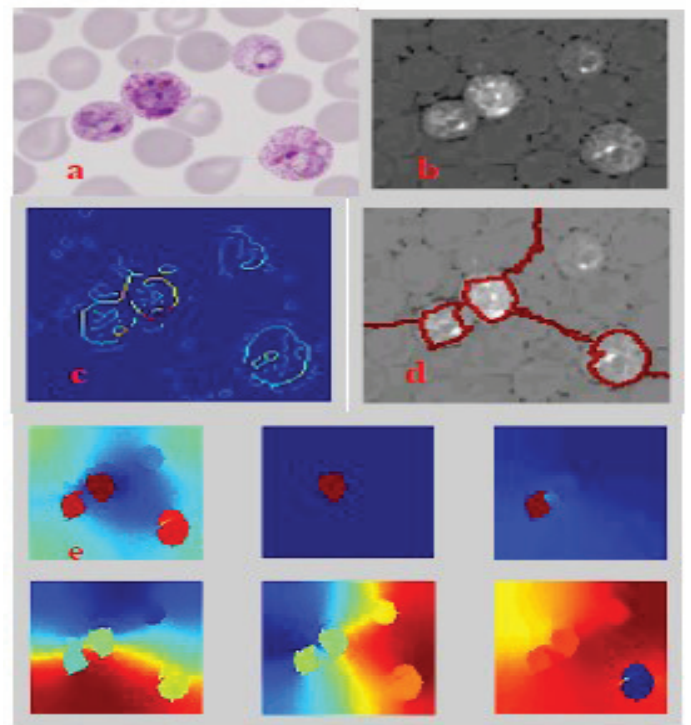

Figure 4. The images * demonstrate segmentation in the HSV color field sing nbsegment=6(a)icroscope image, (b) Brightness image, (c) Edge view, (d) Segment display, (e) Eigenvectors display.

This model is seen as generally appropriate. When all is said in (and) done, HSV is generally utilized for image handling applications however the essential usage of NCut depends on RGB shading space. The outcomes here unmistakably delineate an enhanced presentation of NCut in HSV for minute images.

\section{Extracting Red Blood Cell Mask}

The vast majority of the malaria parasites exist in red platelets, along these lines if red platelets are isolated from different components of blood, the precision and speed of identifying intestinal sickness parasites are improved essentially. We rendered a paired picture to this closing by using Otsu's thresholding. It deserves to refer to the fact that 
the calculation of restriction is derived from the base measure of the inner distinction of the given weight in Otsu's method, and as shown by the critical principle, it follows up on images of grayscale. This technique is a good response for getting to paired images without losing information, particularly in the blood scope tests. To have an ideal parallel picture to suit the item's early tissue, it is necessary to perfect the precision of the matched image with the aggregation of the two-aspect identification images from Canny's approach and the double image from Otsu's approach. At that point, the components which aren't stuffed, because of the absence of exactness in thresholding, can be crammed all over again. Since the discovering crimson platelets within the image are the factor of this method, undesired components that were littler than red platelets be erased through morphology activity. For doing this, a plate shape auxiliary component be utilized [8] $\square$

\section{E. Cell Feature Evaluation and Selection}

When cells have identified as well as portioned from, take an entire image within the subsequent stage, we extricate every sectioned cell and portray them by their shading and surface data to recognize tainted cells from typical cells inside a learning structure. Figure 5 shows instances of separated contaminated and typical cells (first and second lines) for human-NIAID (first section) and mouse-NIAID (second and third segment) data sets.

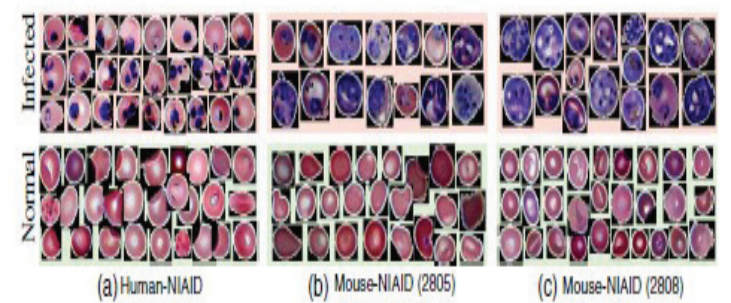

Figure 5. Separated, contaminated and Typical cells

Definitions of contaminated (first row) and regular (second row) cells collected for (a) human-NIAID, (b) mouse-NIAID dataset 2805, and (c) mouse-NIAID 2808.

We read different highlights to represent normal and anomalous cells and analyzed their appearance using SVM and ANN classifiers to pick the most unequal group of components. We analyzed the presentation on both SVM and ANN to prove that the best list of capacities beats different autonomous highlights from the classifier used [25]. In highlight assessment tests, we utilized earth fact explanations toward remove cells along with decoupling exhibition of highlights and classifiers from our programmed division outputs.

\section{RESULTS}

Plasmodium Vivax data set (37 images), includes pictures of both dense and thin blood spreads for all levels of dwelling growth (Trophozoite, Gametocytes, Ookinete, and Schizonts). Our experiments preclude gametocytes and the Ookinete classes since the human body is out of bounds. In the trial, we break the trophozoites (ring configuration and formative levels) and the Schizonts. Figure 6 shows the. Images of diverse tiers of improvement of malaria parasite interior human blood. The formative levels are represented in discern $8(\mathrm{a}-\mathrm{h})$, the division outcomes of the equation are given in parent 10 . The perceptions display that the consequences were given utilizing the HSV shading area gives the most precise outcomes. The facts pix containing 1 four amazing protozoa inflamed cells are notions about this deliberation.

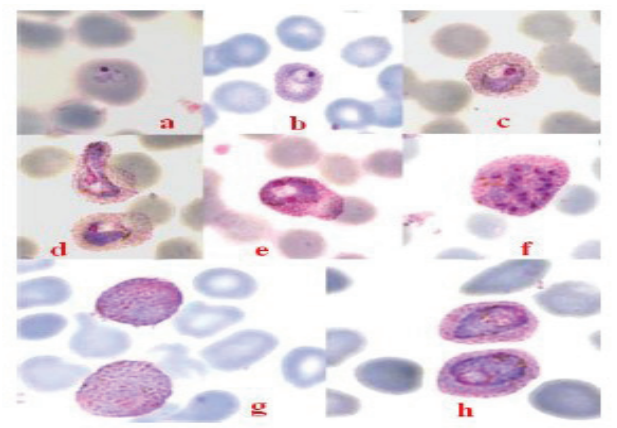

Figure 6. Images of diverse tiers of improvement of malaria parasite interior human blood grabbed using shiny challenge microscope

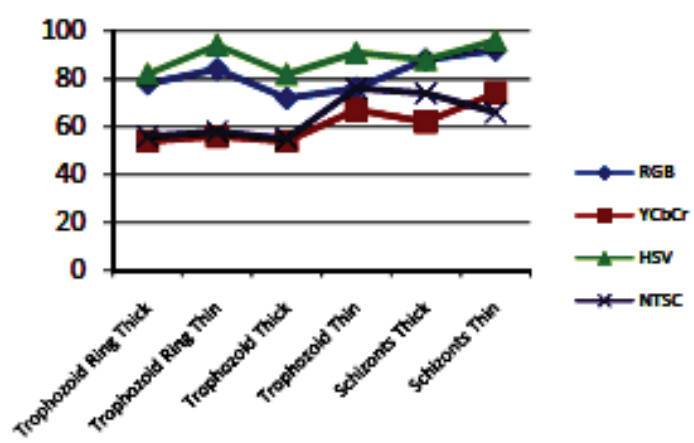

Figure 7. Presentation of segmentation

The proportional presentation of segmentation uses specific color areas on numerous ranges of parasitic proliferation the use of leveled RI's. It is shown in Figure 7. Different proportional segmentation uses different color areas on numerous ranges of parasitic proliferation. The segmentation of corresponding degrees of parasitic proliferation and degrees of improvement of the malaria parasite is shown in Figure 8.

We likewise attempted the calculation on images having diverse overlaying RBCs, comparable yields, comparable effects beneath those situations additionally. The RGB execution appears to move downward by compound multicell sights yet HSV is visible toward increasingly constant regardless of change of cells in the foundation and without malarial cells. Slide pix segmentation is prepared based on and without inflamed RBC's as shown in Figure 9. Recall that the special snapshots, b, e give away the polluted, flamed RBCs while c,f give away the non-inflamed RBCs. 


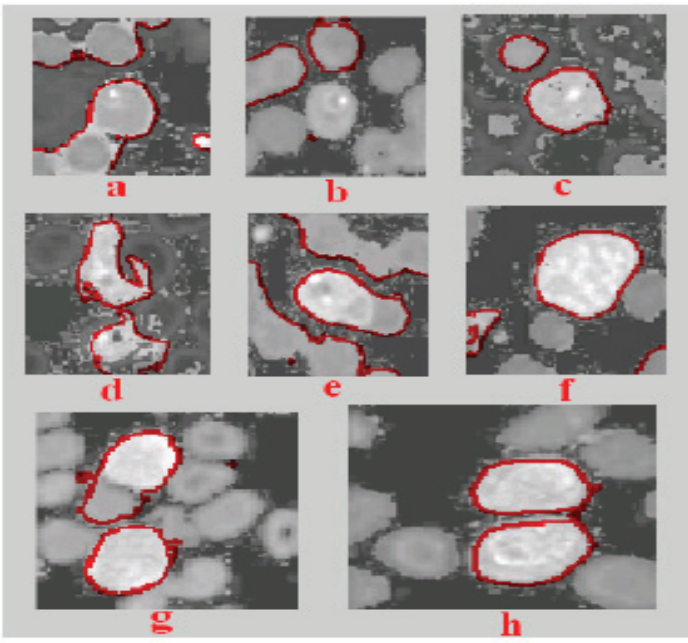

Figure 8 . The segmentation of corresponding degrees of parasitic proliferation almost about numerous degrees of improvement of the malaria parasite

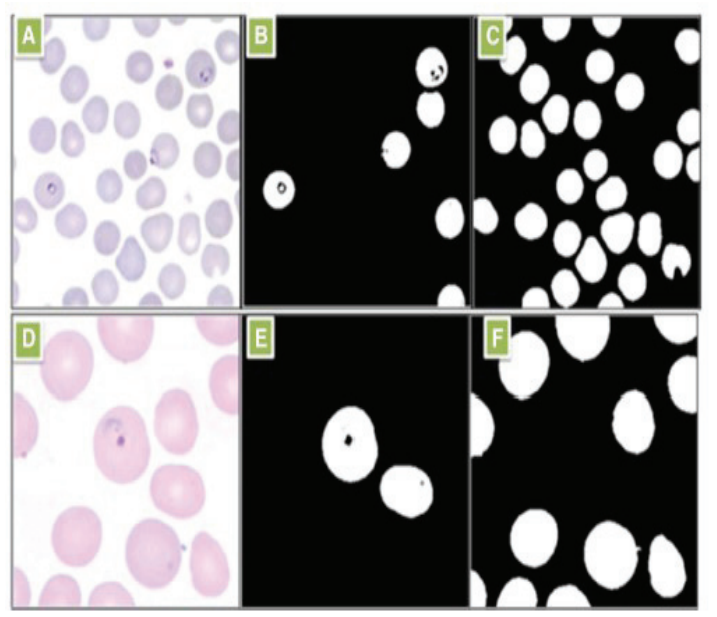

Figure 9. Slide pix segmentation system with inflamed RBCs

\section{CONCLUSIONS}

This paper presents a computationally gifted technique for branch and portrayal of malarial parasites from fringe blood smear images. Outcomes show that NCut calculation is excellent within the HSV shading region. The coloration of the parasites is the principle one in each type a property and this is the equivalent in all moderate blood smear advanced images. In any case, the methodology experiences commotion, however, we tended to it by dropping its impact is twofold totally. The method proposed is here automatic, more precise compared to other available methods, and also rapidly eliminating the human interventions in the diagnosis.

\section{REFERENCES}

1. Sathpathi et al, 2014, "Comparing Leishman and Giemsa staining for the assessment of peripheral blood smear preparations in a malaria-endemic region in India"

2. Walliander $\mathrm{t}$ al, 2012 "Automated segmentation of blood cells in Giemsa stained digitized thin blood films"
3. Katherine Torres, Automated microscopy for routine malaria diagnosis: a field comparison on Giemsa- stained blood films in Peru.

4. Leila Malihi, Malaria Parasite Detection in Giemsa-Stained Blood Cell Images, 2013.

5. Ishan R. Dave, Image Analysis for Malaria Parasite Detection from Microscopic Images of Thick Blood Smear, 2018.

6. Mahdieh Poostchi, Malaria parasite detection and cell counting for human and mouse using thin blood smear microscopy, 2020.

7. Subhamoy Mandal, 2010, Segmentation of blood smear images using normalized cuts for detection of malarial parasites

8. Naveed Abbas, 2016, "Machine aided malaria parasitemia detection in Giemsa-stained thin blood smears".

9. K. Abba, J.J. Deeks, P. Olliaro, C-M. Naing, S.M. Jackson, Y. Takwoingi, S. Donegan, and P. Garner. Rapid diagnostic tests for diagnosing uncomplicated p. falciparum malaria in endemic countries. Cochrane Database Syst Rev, 7, 2011.

10. L. Allen, J. Hatfifield, G. DeVetten, J. Ho, and M. Manyama. Reducing malaria misdiagnosis: the importance of correctly interpreting Para check Pf. BMC infectious diseases, 11(1):308, 2011.

11. B.B. Andrade, A. Reis-Filho, A.M. Barros, S.M. Souza-Neto, L.L. Nogueira, K.F. Fukutani, E.P. Camargo, L.M.A. Camargo, A. Barral, A. Duarte, and M. Barral-Netto. Towards a precise test for malaria diagnosis in the Brazilian Amazon: comparison among field microscopy, a rapid the diagnostic test, nested PCR, and a computational - expert system based on artificial neural networks. $\square$ Malaria Journal, 9:117, 2010.

12. J. Bailey, J. Williams, B.J. Bain, J. Parker-Williams, and P.L. Chiodini. Guideline: the laboratory diagnosis of malaria. British Journal of Hematology, 163(5):573-580, 2013.

13. D.N. Breslauer, R.N. Maamari, N.A. Switz, W.A.Lam, and D.A. Fletcher. Mobile phone-based clinical microscopy for global health applications. PLoS One, 4(7):e6320, 2009.

14. M. Everingham, L. Van Gool, C.K.I. Williams, J. Winn, and A. Zisserman. The PASCAL Visual Object Classes (VOC) Challenge. International Journal of Computer Vision, 88(2):303-338, 2010.

15. P. Geurts, D. Ernst, and L. Wehenkel. Extremely randomized trees. Machine Learning, 63(1):3-42, 2006.

16. H.J.A.M. Heijmans. Morphological Image Operators. Academic Press, Boston, 1994.

17. H.J.A.M. Heijmans. Connected morphological operators for binary images. Computer Vision and Image Understanding, 73:99-120, 1999.

18. Mobile Point-of-Care Monitors and Diagnostic Device Design

19. B. Houwen. Blood film preparation and staining procedures. Laboratory Hematology, 6(1):1-7, 2000.

20. D.S. Ishengoma, F. Francis, B.P. Mmbando, J.P.A. Lusingu, P. Magistrado, M. Alifrangis, T.G. Theander, I.C. Bygbjerg, and M.M. Lemnge.Accuracy of malaria rapid diagnostic tests in community studies and their impact on the treatment of malaria in an area with a declining malaria burden in north-eastern Tanzania. Malaria Journal, 10:176,2011.

21. M. Kiggundu, S.L. Nsobya, M.R. Kamya, S. Filler, S. Nasr, G. Dorsey, and A. Yeka. Evaluation of a comprehensive refresher training program in malaria microscopy covering four districts of Uganda. TheAmerican Journal of TropicaMedicine and Hygiene, 84(5):820, 2011.

22. O.A. Koita, O.K. Doumbo, A. Ouattara, L.K. Tall, A. Konar'e, M. Diakit'e, M. Diallo, I. Sagara, G.L. Masinde, S.N. Doumbo, et al. False-negative rapid diagnostic tests for malaria and deletion of the histidine-rich repeat region of the hrp2 gene. The American Journal of Tropical Medicine and Hygiene, 86(2):194-198, 2012. 
23. V.V. Makkapati and R.M. Rao. Segmentation of malaria parasites in peripheral blood smear images. IEEE International Conference on Acoustics, Speech and Signal Processing, 2009.

24. L.M. Milne, M.S. Kyi, P.L. Chiodini, and D.C. Warhurst. Accuracy of routine laboratory diagnosis of malaria in the United Kingdom. Journal

25. of clinical pathology, 47(8):740-742, 1994.

26. M.G. Mubangizi, C. Ikae, A. Spiliopoulou, and J.A. Quinn. Coupling spatiotemporal disease modeling with the diagnosis. In Proceedings of the International Conference on Artificial Intelligence (AAAI), 2012.
27. C.K. Murray, R.A. Gasser, A.J. Magill, and R.S. Miller. Update on rapid diagnostic testing for malaria. Clinical Microbiology Reviews, 21:97-110, 2008

28. World Health Organization. Malaria light microscopy: creating a culture of quality. In Report of WHO SEARO/WPRO workshop on quality assurance for malaria microscopy, Geneva, 2005.

29. D. Payne. Use and limitations of light microscopy for diagnosing malaria at the primary health care level. Bulletin of the World Health Organization, 66(5):621, 1988. 